\title{
Imobilização de metais pesados presentes nos resíduos de quartzito por meio da incorporação em argamassas com substituição total do agregado natural
}

\author{
Immobilization of heavy metals present in quartzite residues through \\ incorporation in mortars with total substitution of the natural aggregate
}
Sâmea Valensca Alves Barros' ${ }^{1}$, Gerbeson Carlos Batista Dantas ${ }^{1 *} \odot$, Gelmires de Araújo Neves ${ }^{2} \odot$, Romualdo Rodrigues Menezes ${ }^{2}$ ()

\begin{abstract}
RESUMO
Este trabalho objetivou imobilizar metais pesados presentes nos resíduos de quartzito por meio da incorporação em argamassas em substituição total ao agregado natural. Dois tipos de resíduos foram utilizados: os resíduos de fragmentação das aparas (QS) e os do pó de serragem (QP). Os resíduos foram caracterizados por fluorescência de raios $X$, difração de raios $X$ e ensaios de toxidade conforme metodologia da Norma Brasileira (NBR) 10005, da NBR 10006 e do Toxicity Characteristic Leaching Procedure (TCLP) 1311. Então, foram analisadas composições de argamassas contendo cimento, cal e os resíduos (QS, QP) utilizando-se a técnica de delineamento de mistura, determinando os melhores traços, o melhor tipo de cura (imersa ou úmida) e, posteriormente, avaliando a resistência dos corpos de prova após cura. A caracterização do resíduo de quartzito revelou a presença de vários metais pesados e extratos lixiviados em contato com a água, classificando-o como resíduo de Classe I - Perigoso. Os resultados indicam que as composições determinadas foram capazes de imobilizar os metais pesados presentes nos resíduos de quartzitos, bem como resultou em argamassas com resistências mecânicas superiores a $2 \mathrm{MPa}$. Por fim, mostrou-se que essa técnica de solubilização e estabilização dos contaminantes presentes nos resíduos de quartzito é uma alternativa tecnologicamente viável e ambientalmente adequada para a destinação final desses resíduos.
\end{abstract}

Palavras-chave: rochas ornamentais; imobilização de contaminantes; resíduos classe l; resíduos perigosos.

\begin{abstract}
This work aims to immobilize heavy metals present in the quartzite residues by means of the incorporation in mortars in total substitution to the natural aggregate. Two types of residues were used: quartzite sand (QS) and powder (QP). The residues were characterized by $x$-ray fluorescence, $x$-ray diffraction, and toxicity tests according to the methodology of the NBR 10005, 10006 and Toxicity Characteristic Leaching Procedure (TCLP) 1311. Then, compositions of mortar containing cement, lime and residues (QS, QP) were analyzed using technique of designing the mixture, determining the best mixture, the best type of cure (immersed or wet), and subsequently evaluated at resistance of the body of evidences after curing. The characterization of quartzite residue revealed the presence of multiple heavy metals and leached extracts in contact with the water, classifying it a Class I-Hazardous waste. Regarding the production of mortars, the results indicated that the compositions determined were able to immobilize the heavy metals present in the quartzite residues, as well as resulted in mortars with mechanical resistances higher than $2 \mathrm{MPa}$. Finally, it was shown that this technique of solubilization and stabilization of contaminants present in quartzite residues is a technologically viable and environmentally adequate alternative for the final destination of these residues.
\end{abstract}

Keywords: ornamental rocks; immobilization of contaminants; class I waste; hazardous waste.

'Universidade Federal Rural do Semi-Árido - Angicos (RN), Brasil.

2Universidade Federal de Campina Grande - Campina Grande (PB), Brasil.

*Autor correspondente: gerbeson_dantas@hotmail.com

Recebido: 25/07/2018 - Aceito: 26/08/2019 - Reg. Abes: 20180063 


\section{INTRODUÇÃO}

No mundo, a geração de resíduos sólidos oriundos do corte e da serragem de rochas ornamentais tem sido cada vez mais problemática, tanto pela geração de vultosos volumes de resíduos quanto pela destinação final ambientalmente inadequada destes (MOREIRA; MANHÃES; HOLANDA, 2008; ERCIKDI; KULEKCI; YILMAZ, 2015). Esses resíduos são materiais não biodegradáveis que, quando destinados inadequadamente no meio ambiente, promovem a degradação dos sistemas naturais, contaminando os corpos hídricos e o solo, além de provocar desequilíbrio dos sistemas ambientais naturais e prejudicar a saúde da população (RIBEIRO et al., 2007; MERSONI; REICHERT, 2017; PIMENTEL et al., 2018).

Frequentemente, os resíduos gerados da exploração de rochas ornamentais são classificados pela Associação Brasileira de Normas Técnicas (ABNT) 10004 como resíduos de Classe II B - Inertes (ABNT, 2004a). Entretanto, com o aprofundamento dos estudos desse material, observou-se que parte desses resíduos tem elevado nível de toxidade, isto é, apresenta metais pesados e fluoretos em sua composição, nos extratos superiores ao limitante estabelecido pelo Anexo $\mathrm{F}$ da Norma Brasileira (NBR) 10004, caracterizando-os como resíduos de Classe A - Perigosos (ABNT, 2004a).

Entre os componentes tóxicos encontrados nesses resíduos, destacam-se o arsênio (As), o cromo (Cr), o cádmio (Cd), entre outros, e por essa razão, não devem ser destinados sem o devido controle, à medida que possuem potencial de serem lixiviados, liberando metais pesados para o meio ambiente (SULLIVAN et al., 2010; JUNKES et al., 2012; BRAGA; BRITO; VEIGA, 2012; ARUKWE; EGGEN; MÖDER, 2012; SHARMA et al., 2014). Nesse sentido, vários trabalhos destacam os efeitos adversos desses elementos na saúde humana, bem como nos sistemas ambientais (NAKWANIT et al., 2011; COUSSY et al., 2012; TOUJAGUEZ et al., 2013; AGRAFIOTI; KALDERIS; DIAMADOPOULOS, 2014).

Na contramão dessa discussão, os países emergentes, nos quais está incluído o Brasil, apresentam dificuldade para elaborar e implementar sistemas de gerenciamento de resíduos eficazes e eficientes, apesar das discussões amplamente debatidas nos últimos decênios, promovidas pelas conferências ambientais e pelos documentos legais resultantes destas (DANTAS et al., 2017). Com efeito, apesar do arcabouço legal norteador da política ambiental brasileira, no que concernem os resíduos sólidos, como a Política Nacional do Meio Ambiente (Lei Federal no 6.938/1981), a Constituição Federal de 1988, a Política Nacional de Resíduos Sólidos (Lei Federal n 12.305/2010) e as NBRs da série 10.000, o Brasil permanece em uma posição de timidez quanto às ações concretas de enfretamento dessa problemática (BRASIL, 1981; 1988; 2010).

Nesse contexto desafiador, vários autores convergem pela necessidade de tornar a exploração das rochas ornamentais ambientalmente sustentável e segura. Para isso, a saída fortemente defendida é desenvolver tecnologias ambientalmente adequadas, tecnologicamente exequíveis e economicamente viáveis, baseado nos documentos legais brasileiros, visando ampliar os processos do ciclo de gerenciamento adequado desses resíduos (TORRES et al., 2009; BACARJI et al., 2013; ANDRÉ et al., 2014; TENNICH; KALLEL; OUEZDOU, 2015).

Assim, o gerenciamento adequado dos resíduos de rochas ornamentais agrega valor econômico e ambiental ao produto final. Em adição a isso, é uma excelente oportunidade de mercado, visto que promove a diversificação da produção, gerando matérias-primas alternativas para vários setores industriais, além de adequar as empresas à legislação ambiental brasileira (JIMÉNEZ et al., 2013; GUILLÉN et al., 2014; GAMEIRO; BRITO; SILVA, 2014; SUKHOLTHAMAN; SHIRAHADA, 2015; SCHACKOW et al., 2015). Nessa perspectiva, a produção de matérias-primas alternativas tem ganhado notoriedade em função do seu consumo cada vez maior. Segundo Camarini e Soares (2018), o consumo de areia para a construção civil alcançou a incrível marca de 391 milhões de toneladas, com tendência de crescimento cada vez mais acentuada.

Entre as rochas ornamentais mais consumidas no mundo, estão as pedras de quartzito. Essas rochas vêm sendo muito utilizadas como pedras ornamentais em elementos decorativos de fachadas, em pisos e, recentemente, como alternativa ao granito, particularmente em bancadas. O quartzito é uma rocha metamórfica constituída de cristais de quartzo imersos em uma matriz cimentante rica em sílica. No seu processamento para produção de itens ornamentais há a produção de dois tipos de resíduos. O primeiro é gerado na forma de pó, em razão da serragem da rocha para produção de placas de quartzito. E o segundo é gerado na forma de retalhos de placas, originados no processamento das placas de quartzito.

Com base nas características do quartzito, acredita-se que o seu resíduo possa ser usado como matéria-prima alternativa na produção de argamassas. Estudos vêm destacando que a incorporação de resíduos de rochas ornamentais em argamassas e concretos é uma alternativa tecnologicamente viável. Patel e Shah (2015), Ergün (2011) e Hameed e Sekar (2009) estudaram a incorporação de resíduos de rochas ornamentais em concreto como forma de reutilização desses resíduos. Mármol et al. (2010) e Ramos et al. (2013) usaram resíduos de granito (rocha ornamental) em argamassas como substituto parcial do cimento, enquanto Martínez et al. (2013) estudaram os resíduos de granito como material de enchimento e de pigmentação em argamassas.

No entanto, os estudos recentes não têm considerado o comportamento de lixiviação dos resíduos, o seu potencial toxicológico após incorporação nos compósitos de matriz cimentícia e o potencial de imobilização dos metais pesados presentes nos resíduos desses materiais. Ademais, não se observam estudos na literatura mundial voltados para a reutilização do resíduo do quartzito por meio de sua incorporação em argamassas para uso na construção civil. 
Assim, frente o enorme volume de resíduo gerado na produção do quartzito, de sua importância econômica e da falta de estudos envolvendo a reutilização desses resíduos, esta pesquisa teve como objetivo imobilizar metais pesados presentes nos resíduos de quartzito por meio da incorporação em argamassas em substituição total ao agregado natural. Para isso, foram determinadas as composições de argamassa que têm boa resistência mecânica, que permitam imobilizar o As e outros metais, bem como verificar a condição de cura que proporciona melhor desempenho mecânico para realizar o estudo do lixiviado das argamassas produzidas.

\section{METODOLOGIA}

\section{Materiais}

Os materiais utilizados nesta pesquisa foram: Cimento Portland (Nassau' ${ }^{\circ}, \mathrm{CP}$ IV $32 \mathrm{RS}$ ), cal hidratada (Carbomilò, Brazil) e resíduos de quartzito. Os resíduos foram coletados em empresas do Brasil que beneficiam e comercializam rochas ornamentais, sendo de dois tipos: na forma de pó, designado QP, proveniente da serragem das placas de quartzito; e na forma de areia, denominado QS, oriunda da cominuição das aparas originadas no processamento das placas de quartzito.

\section{Caracterização química, mineralógica e ambiental dos resíduos de quartzito}

Para caracterização do resíduo de quartzito, utilizaram-se as seguintes técnicas: fluorescência de raios X (equipamento EDX 720 da Shimadzu) e difração de raios X (equipamento XRD 6000 da Shimadzu) com varredura entre 5 e $80^{\circ}$ e velocidade angular de $1,2 \mathrm{deg} / \mathrm{min}$.

A caracterização ambiental ocorreu de acordo com a realização das análises químicas dos extratos lixiviados e solubilizados por absorção atômica (modelo AA-7000, ar- $\mathrm{C}_{2} \mathrm{H}_{2}$ ), utilizando como parâmetros As, alumínio (Al), bário $(\mathrm{Ba}), \mathrm{Cd}$, cromo $(\mathrm{Cr})$, cobre $(\mathrm{Cu})$, ferro $(\mathrm{Fe})$, manganês $(\mathrm{Mn})$, níquel (Ni) e zinco $(\mathrm{Zn})$. Os testes de lixiviação e solubilização foram realizados conforme a NBR 10005 (ABNT, 2004b), a NBR 10006 (ABNT, 2004c) e o Toxicity Characteristic Leaching Procedure (TCLP) 1311 (USEPA, 1992).

\section{Planejamento experimental}

Neste estudo, utilizou-se a técnica do delineamento de mistura para realização do planejamento experimental e determinação das composições das argamassas com resíduos de quartzito que apresentem resistência mecânica adequada para uso na construção civil. No delineamento, foram usados como matérias-primas: o cimento, a cal, os QS e os QP.

$\mathrm{O}$ delineamento de misturas foi definido utilizando-se as seguintes restrições nas formulações: quantidade máxima de cimento de $50 \%$, quantidade máxima de cal de 50\%, quantidade mínima de QS de $50 \%$ e quantidade máxima de QP de 15\%. As restrições basearam-se em dados da literatura e características de processamento das formulações. Buscou-se nas formulações substituir totalmente o agregado miúdo convencional (areia) por resíduo de areia de quartzito (QS) e material pulverulento (QP). O delineamento apresentou 21 composições e foi executado com duplicatas conforme a Tabela 1. Na análise, foi utilizado o software Statisca7.

Tabela 1 - Composições (em \% mássica) obtidas no delineamento de misturas para formulação das argamassas com resíduos de quartzito e resistência à compressão simples aos 28 dias, determinadas após cura úmida e cura imersa.

\begin{tabular}{|c|c|c|c|c|c|c|c|c|c|c|}
\hline & \multirow{2}{*}{ Cimento } & \multirow{2}{*}{ Cal } & \multirow{2}{*}{ QS } & \multirow{2}{*}{ QP } & \multicolumn{3}{|c|}{ CS-UC (MPa) } & \multicolumn{3}{|c|}{ CS-IC (MPa) } \\
\hline & & & & & 1 & 2 & 3 & 1 & 2 & 3 \\
\hline 1 & 0 & $\mathrm{O}$ & 100 & 0 & 0 & 0 & 0 & 0 & $\mathrm{O}$ & 0 \\
\hline 2 & 50 & 0 & 50 & 0 & 25,96 & 20,87 & 22,40 & 27,48 & 19,86 & 22,40 \\
\hline 3 & 0 & 50 & 50 & 0 & 0,08 & 0,12 & 0,05 & 0,08 & 0,13 & 0,05 \\
\hline 4 & 0 & 0 & 85 & 15 & 0 & 0 & 0 & 0 & 0 & 0 \\
\hline 5 & 35 & 0 & 50 & 15 & 21,40 & 19,44 & 17,73 & 19,08 & 17,94 & 20,52 \\
\hline 6 & 0 & 35 & 50 & 15 & 0,15 & 0,13 & 0,70 & 0,15 & 0,13 & 0,70 \\
\hline 7 & 0 & 0 & 92 & 8 & 0 & 0 & 0 & 0 & 0 & 0 \\
\hline 8 & $\mathrm{O}$ & 25 & 75 & $\mathrm{O}$ & 0,09 & 0,13 & 0,07 & 0,09 & 0,13 & 0,07 \\
\hline 9 & 0 & 42,5 & 50 & 7,5 & 0,14 & 0,21 & 0,10 & 0,14 & 0,21 & 0,10 \\
\hline 10 & 0 & 17,5 & 67,5 & 15 & 0,22 & 0,18 & 0,20 & 0,22 & 0,18 & 0,20 \\
\hline 11 & 25 & 0 & 75 & 0 & 14,55 & 14,25 & 12,56 & 13,70 & 12,23 & 12,43 \\
\hline 12 & 42,5 & 0 & 50 & 7,5 & 21,32 & 18,51 & 21,50 & 20,31 & 19,76 & 20,33 \\
\hline
\end{tabular}


Tabela 1 - Continuação.

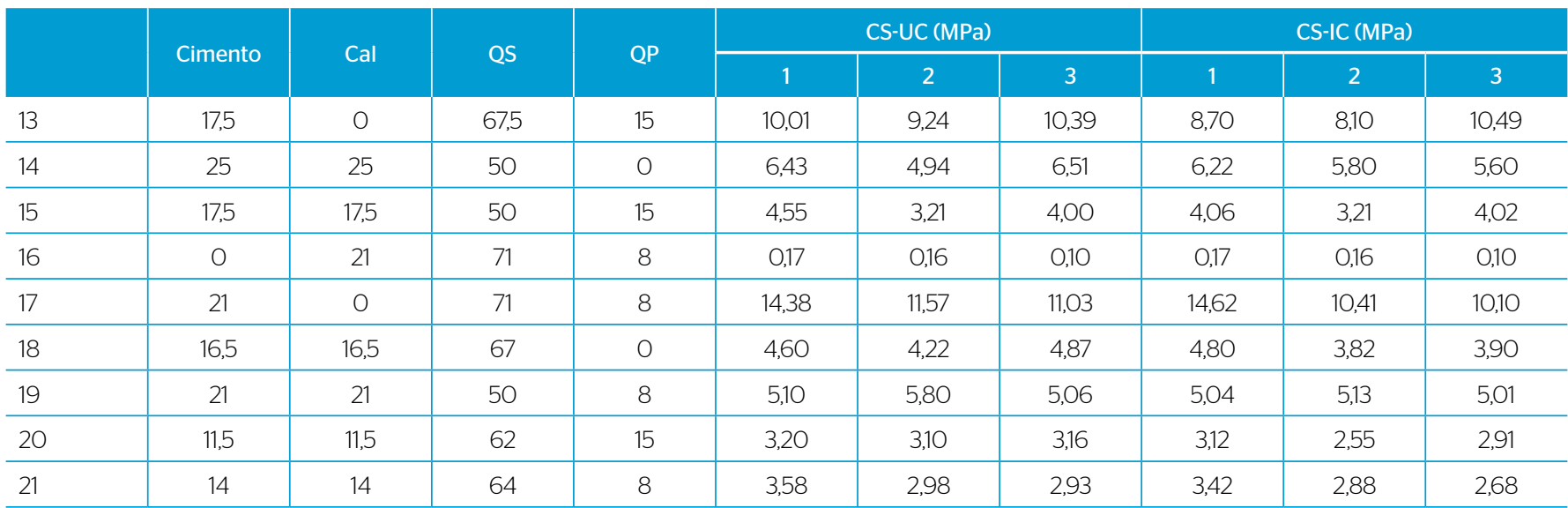

QS: resíduos de fragmentação das aparas; QP: resíduos do pó de serragem; CS-UC: compressão simples por via úmida; CS-IC: compressão simples por via imersa.

Após a formulação do planejamento experimental de delineamento de misturas (Tabela 1), foi determinada a quantidade de água para cada uma das composições, de forma a obter um espalhamento padrão em todas elas, conforme metodologia da ASTM C230 (ASTM, 2008). O espalhamento padrão adotado foi de $260 \pm 10 \mathrm{~mm}$, determinado por meio do ensaio flow table, conforme a NBR 13276 (ABNT, 2002), a fim de garantir adequada trabalhabilidade às argamassas.

\section{Confecção, cura dos corpos de prova e potencial toxicológico das argamassas incorporadas com os resíduos de quartzito}

Os corpos de prova foram produzidos em misturador mecânico com a quantidade de água determinada previamente, sendo moldados cinco corpos de provas de $50 \times 100 \mathrm{~mm}$ para cada composição. Cada valor utilizado no delineamento de mistura corresponde ao valor médio de cinco corpos de prova. Após conformação, os corpos foram curados por via úmida (UC) e por via imersa (IC). A câmara úmida foi confeccionada em caixa de isopor contendo areia úmida, em que os corpos de prova foram envoltos por plástico e enterrados na areia úmida por um período de 28 dias. Já a câmara imersa ocorreu em caixa de isopor com água saturada com $2 \%$ de cal, em que os corpos de prova foram imersos por um período de 28 dias. A resistência à compressão simples (CS) foi determinada após 28 dias em máquina universal de ensaios mecânicos (SHIMADZU AG-IS), conforme NBR 7215 (ABNT, 1996).

Os corpos de prova de argamassas, após curados por 28 dias, foram rompidos e depois destorroados para serem submetidos aos ensaios de lixiviação e solubilização, conforme a NBR 10005 (ABNT. 2004a) e a NBR 10006 (ABNT, 2004b). A análise química dos extratos lixiviados e solubilizados ocorreu utilizando os mesmos parâmetros, metodologia e equipamentos adotados com os resíduos de quartzito.

\section{RESULTADOS E DISCUSSÃO}

\section{Caracterização química, mineralógica e ambiental do resíduo de quartzito}

A composição química dos QS e dos QP está apresentada na Tabela 2. Os resíduos apresentaram valores elevados de sílica e alumina por terem como protólitos arenitos quartzosos e chertsilicosos. Os teores de sílica $(67,50$ e 77,90\%) demonstram o caráter quartzoso das amostras, enquanto os teores de alumina (17,28 e 11,91\%) ocorrem em razão da presença de feldspato e mica, embora em menor quantidade que o quartzo.

Observa-se também a presença de óxido de potássio, óxido de ferro, óxido de magnésio e óxido de cálcio nas amostras, comportando-se como minerais acessórios, já que possuem teores inferiores a 5\%, com exceção do óxido de potássio. Os demais óxidos apresentaram valores abaixo de $1 \%$ (Tabela 2).

Os difratogramas de raios $\mathrm{X}$ das amostras de quartzito (QS e QP) podem ser vistos na Figura 1. Observaram-se as seguintes fases mineralógicas presentes no resíduo de quartzito: quartzo, caracterizado pela distância interplanar 3,33 Å, que é um mineral inerte por ser cristalino; caulinita $\left(\mathrm{Al}_{2} \mathrm{Si}_{2} \mathrm{O}_{5}(\mathrm{OH})_{4}\right)$, caracterizada pelas distâncias interplanares 7,15 e 3,71 $\AA$; feldspato $\left(\mathrm{KSi}_{3} \mathrm{AlO}_{3}\right)$, caracterizado pela distância interplanar 3,45 ̊, que são feldspatos alcalinos ricos em íons $\mathrm{K}^{+}$e água $\mathrm{H}^{+}$- esses íons $\mathrm{H}^{+}$, quando tomam o lugar dos íons $\mathrm{K}^{+}$, são liberados para os íons livres de água e, em menor quantidade, para a micabiotita $\mathrm{K}(\mathrm{Mg}, \mathrm{Fe})_{3}(\mathrm{OH}, \mathrm{F})_{2}(\mathrm{Al}, \mathrm{Fe}) \mathrm{Si}_{3} \mathrm{O}_{10}$, caracterizada pelas distâncias interplanares 9,72 e 4,98 ̊̊.

Os resultados obtidos nos difratogramas permitem constatar que os resíduos de quartzito são constituídos principalmente de minerais de quartzo, seguidos por caulinitas, feldspatos e, em menor quantidade, micas, corroborando com os resultados da análise química. A reflexão 
mais intensa e cristalina ocorre no intervalo $25^{\circ}<2 \theta<30^{\circ}$, característica do quartzo, justificando o percentual predominante de $\mathrm{SiO}_{2}$ nas amostras. A presença da alumina é explicada predominantemente pela caulinita e pelo feldspato, mas como as reflexões apresentaram baixa intensidade, justifica o teor relativamente baixo de alumina nas

Tabela 2 - Composição química dos resíduos de fragmentação das aparas e dos resíduos do pó de serragem.

\begin{tabular}{l|c|c} 
Composição química (\%) & QS & QP \\
\hline $\mathrm{SiO}_{2}$ & 67,50 & 77,90 \\
\hline $\mathrm{Al}_{2} \mathrm{O}_{3}$ & 17,28 & 11,91 \\
\hline $\mathrm{K}_{2} \mathrm{O}$ & 7,22 & 4,83 \\
\hline $\mathrm{Fe}_{2} \mathrm{O}_{3}$ & 2,20 & 1,22 \\
\hline $\mathrm{MgO}$ & 1,62 & 0,93 \\
\hline $\mathrm{CaO}$ & 1,20 & 0,83 \\
\hline $\mathrm{SO}$ & 0,36 & 0,28 \\
\hline $\mathrm{BaO}$ & 0,21 & 0,24 \\
\hline Perda ao fogo & 1,997 & 1,605 \\
\hline
\end{tabular}

QS: resíduos de fragmentação das aparas; QP: resíduos do pó de serragem. amostras. Os baixos teores de $\mathrm{MgO}$ e $\mathrm{CaO}$ são provenientes do carbonato de magnésio e da calcita fornecidos pela pequena presença de dolomita. O maior percentual de potássio e ferro na amostra QS deve-se à maior intensidade da reflexão principal característica da mica, que ocorre no intervalo $5^{\circ}<2 \theta<10^{\circ}$, em detrimento da amostra QP, que possui a reflexão característica da mica, porém com baixa intensidade.

A Tabela 3 evidencia o extrato lixiviado e solubilizado dos resíduos QS e QP. Os extratos lixiviados e solubilizados dos resíduos de quartzito foram analisados quanto às concentrações de $\mathrm{Al}, \mathrm{As}, \mathrm{Ba}, \mathrm{Cd}, \mathrm{Cr}$, $\mathrm{Cu}, \mathrm{Fe}, \mathrm{Mn}$, Ni e Zn, conforme indicado no Anexo F da NBR 10004 (ABNT, 2004a), e indicaram a presença desses elementos em desacordo com os teores máximos permitidos pelo TCLP 1311 (USEPA, 1992), pela NBR 10005 (ABNT, 2004b) e pela NBR 10006 (ABNT, 2004c). De acordo com os resultados, a amostra QS apresentou como contaminantes: As (5,96 vezes superior ao valor máximo permitido), $\mathrm{Al}$ (24,2 vezes superior ao valor máximo permitido), Cd (0,26 vez superior ao valor máximo permitido), $\mathrm{Fe}$ (5,57 vezes superior ao valor máximo permitido) e Mn (3,68 vezes superior ao valor máximo permitido). Já a amostra QP apresentou os seguintes contaminantes:
A

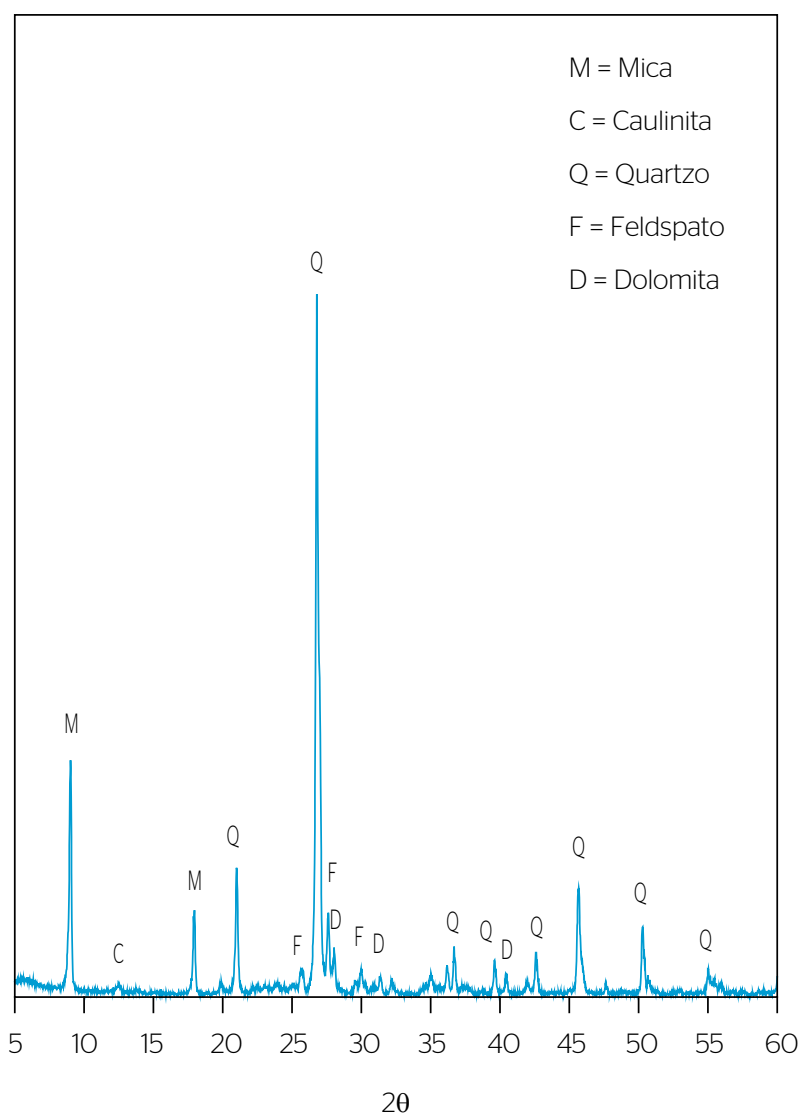

B

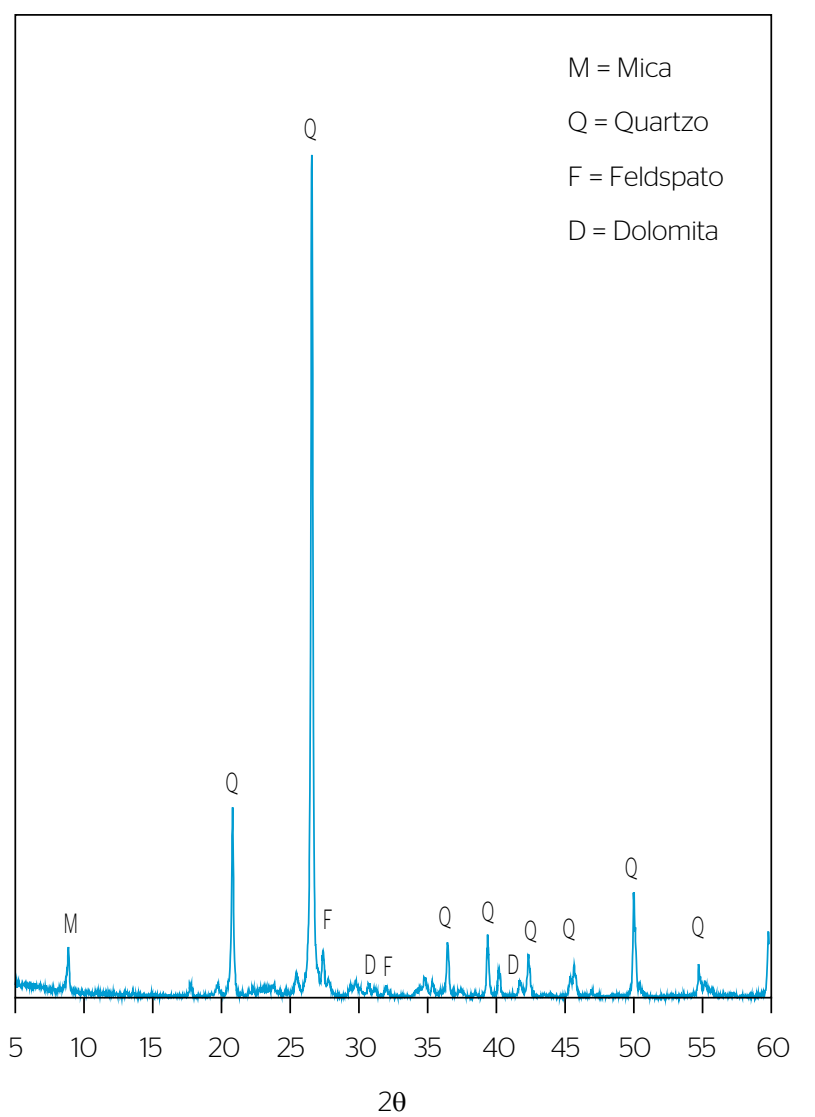

Figura 1 - Difratogramas de raios $X$ das amostras de (A) fragmentação das aparas e (B) pó de serragem. 
Tabela 3 - Teores determinados e máximos permitidos para o extrato lixiviado e solubilizado das amostras de resíduos de fragmentação das aparas e de resíduos do pó de serragem.

\begin{tabular}{|c|c|c|c|c|c|c|}
\hline \multirow{3}{*}{ Parâmetro } & \multicolumn{3}{|c|}{ Lixiviação } & \multicolumn{3}{|c|}{ Solubilização } \\
\hline & \multicolumn{2}{|c|}{ Teores determinados } & \multirow{2}{*}{$\begin{array}{c}\text { Teores máximos } \\
\text { permitidos (mg/L) }\end{array}$} & \multicolumn{2}{|c|}{ Teores determinados } & \multirow{2}{*}{$\begin{array}{c}\text { Teores máximos } \\
\text { permitidos (mg/L) }\end{array}$} \\
\hline & QS & QP & & QS & $\mathrm{QP}$ & \\
\hline $\mathrm{Al}$ & 4,840 & 39,700 & 0,200 & 4,700 & 0,200 & 0,200 \\
\hline As & 29,800 & 38,500 & 5,000 & 19,80 & 26,700 & 0,010 \\
\hline $\mathrm{Ba}$ & 0,450 & 1,200 & 70,000 & 0,370 & 0,080 & 0,700 \\
\hline $\mathrm{Cd}$ & 0,130 & 0,010 & 0,500 & 0,135 & $<0,050$ & 0,005 \\
\hline $\mathrm{Cr}$ & 0,220 & 0,050 & 5,000 & 0,220 & 0,010 & 0,050 \\
\hline $\mathrm{Cu}$ & 0,050 & 2,540 & 2,000 & 0,050 & 0,020 & 2,000 \\
\hline $\mathrm{Fe}$ & 1,670 & 5,340 & 0,300 & 1,670 & 0,010 & 0,300 \\
\hline $\mathrm{Ni}$ & 0,030 & 1,170 & 7,000 & 0,030 & $<0,010$ & 0,070 \\
\hline $\mathrm{Mn}$ & 1,470 & 3,870 & 0,400 & 1,460 & 0,010 & 0,100 \\
\hline $\mathrm{Zn}$ & 0,230 & 0,530 & 5,000 & 0,190 & 0,020 & 5,000 \\
\hline
\end{tabular}

QS: resíduos de fragmentação das aparas; QP: resíduos do pó de serragem.

As (7,7 vezes superior ao valor máximo permitido), $\mathrm{Al}$ (198,5 vezes superior ao valor máximo permitido), $\mathrm{Cu}$ (1,27 vez superior ao valor máximo permitido), Fe (17,8 vezes superior ao valor máximo permitido) e Mn (9,68 vezes superior ao valor máximo permitido). Os maiores teores de contaminantes foram observados na amostra QP. Por fim, com base nos resultados encontrados no extrato lixiviado (Tabela 3), ambos os resíduos de quartzito são classificados como resíduo de Classe I - Perigoso em razão do caráter tóxico, conforme estabelecido pela NBR 10004 (ABNT, 2004a).

Os resultados da Tabela 3 destacam a necessidade de reutilizar os resíduos de quartzito, tendo como maior prioridade a imobilização dos metais pesados contidos nos resíduos, e não apenas a quantidade adicionada à argamassa ou sua pura resistência mecânica, como tem sido observado na literatura. Logo, torna-se necessário utilizar traços que permitam esse processo. Segundo Gerven et al. (2004), os resíduos sólidos perigosos que apresentam metais pesados em sua constituição, quando reutilizados em matrizes cimentícias, podem ter a lixiviação dos metais pesados controlada. Os autores advogam que a carbonatação da portlandita $\left(\mathrm{Ca}(\mathrm{OH})_{2}\right)$ e/ou do silicato hidratado de cálcio (C-S-H), pelo gás carbônico, promove a alteração da solubilidade dos componentes e da porosidade da matriz, reduzindo a lixiviação dos metais pesados.

\section{Resistência à compressão simples das composições de argamassas determinadas no delineamento de misturas e melhor condição de cura}

Com base nos dados obtidos na Tabela 1, determinaram-se as equações de regressão (Equações 1 e 2), que descrevem o comportamento da resistência à CS aos 28 dias em função das proporções de cimento, cal, QS e QP para cada condição de cura ao nível de 5\% de significância.

CS-UC $=28,59$ cimento $-0,258 \mathrm{cal}+0,217 \mathrm{QS}+16,69 \mathrm{QP}+$ 164,13 cimento cal $+34,12$ cimento $\mathrm{QS}+37,59$ cimento QP $+0,48 \mathrm{cal}$ QS - 11,06 cal QP - 22,74 QS QP - 494,77 cimento cal QS - 649,68 cimento cal QP

Em que:

CS-UC = compressão simples por via úmida;

QS = resíduos de fragmentação das aparas;

$\mathrm{QP}=$ resíduos do pó de serragem.

CS-IC $=36,58$ cimento $-0,298 \mathrm{cal}+0,196 \mathrm{QS}+13,45 \mathrm{QP}+$ 143,15 cimento cal $+18,63$ cimento QS $+31,95$ cimento QP + 0,58 cal QS - 7,4 cal QP - 18,78 QS QP - 458,92 cimento cal QS - 628,26 cimento cal QP

Em que:

CS-IC = compressão simples por via imersa;

QS = resíduos de fragmentação das aparas;

$\mathrm{QP}=$ resíduos do pó de serragem.

Os modelos de regressão (Equações 1 e 2) usados neste estudo são estatisticamente significativos no nível requerido e apresentam pouca variabilidade (altos coeficientes de determinação múltipla). Os coeficientes de determinação múltipla indicam a porcentagem de variação na resistência à compressão que é explicada pela variação deliberada 
das frações de matéria-prima no estudo. A significância dos modelos derivados também pode ser avaliada comparando o valor do teste $\mathrm{F} \mathrm{e}$ o valor $\mathrm{F}$ tabulado na distribuição de Fisher-Snedecor. As regressões são consideradas estatisticamente significativas, ou seja, as flutuações em razão das variáveis independentes são explicadas principalmente pelo modelo, se os valores de $\mathrm{F}$ forem maiores que os valores tabulados. Os valores de F relacionados às Equações 1 e 2 são mais de cinco vezes superiores aos valores tabelados.

As principais propriedades estatísticas das regressões obtidas com a análise de variância ao nível de 5\% (Tabela 4), utilizando a nomenclatura usualmente relatada na literatura, mostram que o modelo mais significativo e adotado em função do valor de $\mathrm{R}^{2}$ e do valor $\mathrm{p}$ (tem que ser inferior ao nível significância) foi a cúbica, tanto para UC quanto para IC.

Com base nos resultados, percebe-se que os modelos lineares e quadráticos apresentaram também excelentes valores de $\mathrm{R}^{2}$, tanto para UC quanto para IC, porém o teste $\mathrm{F}$ determina a significância e a realização das previsões de um modelo. Segundo Box e Wetz (1973), um modelo estatístico é significativo e preditivo quando o valor do F calculado é, no mínimo, quatro a cinco vezes o valor de F tabelado em distribuição Fisher-Snedecor. Logo, analisou-se o valor do teste F obtido e o F tabelado em distribuição Fisher-Snedecor para verificar qual é o melhor modelo e, consequentemente, a melhor condição de cura, uma vez que esta produz efeitos importantes sobre as propriedades mecânicas, como a resistência à CS, e causa influência na durabilidade das argamassas (SAJEDI; RAZAK, 2011).

Neste trabalho, obteve-se, para UC, F calculado de 17,81 e, para IC, F calculado de 12,33. Logo, a melhor condição de cura é a UC, pois apresenta F calculado maior que o da IC e mais elevado (oito vezes) que o F tabulado. Por outro lado, ao se analisar as Equações 1 e 2, observa-se que a UC propiciou valores de resistência mecânica levemente superiores aos obtidos com a IC em uma faixa de teores de resíduo de quartzito, seja na forma de areia, seja na forma de pó - o que permite incorporar maiores teores de resíduos de quartzito. Esses resultados corroboram com a concepção de Sajedi e Razak (2011) sobre a condição de cura ser responsável por promover a hidratação do cimento em argamassas, causando efeitos positivos na resistência delas.

As superfícies de resposta geradas para resistência à CS para as condições de UC (Figura 2) e de IC (Figura 3), para teores de 0 e 15\% de QP, evidenciam que ambas as condições de cura propiciaram valores de resistência à compressão semelhantes. A cura, segundo Mehta e Monteiro (2008), é definida como o conjunto de reações que ocorrem ao longo do endurecimento e envelhecimento de uma argamassa, podendo condicionar o modo como a água se liberta dela e esta, por sua vez, condicionar as reações de hidratação do cimento, levando à maior resistência, que é um indicador da durabilidade das argamassas. Isso indica que na faixa de composições estudadas os mecanismos de reação envolvendo as curas devem ser semelhantes.

Apesar de a UC ter apresentado valores de resistência à CS melhores que a IC, pode-se perceber que a IC proporciona um efeito positivo em relação à resistência à CS (Figura 3 ).

Resultados semelhantes foram encontrados por Mármol et al. (2010) para resíduos de granito. Eles incorporaram 5, 10 e 20\% de pó de granito, obtendo resistência à CS aos 28 dias mínima de $2 \mathrm{MPa} \mathrm{e}$ máxima de $5 \mathrm{MPa}$, e concluíram que o ideal seria a incorporação de 10\% do resíduo. Já Ramos et al. (2013) também utilizaram 10\% de pó de granito como substituição parcial do cimento e não obtiveram bons resultados no que diz respeito à resistência à CS aos 28 dias, pois ocorreu uma redução no valor quando comparada à da argamassa de referência. Corinaldesi, Moriconi e Naik (2010) obtiveram resultados parecidos para resíduos de mármore, substituindo $10 \%$ da areia por pó de mármore. Porém, para alcançarem bons valores de resistência à CS a 28 dias, tiveram de utilizar um superplastificante na mistura.

Embora o uso desses resíduos por esses autores não tenha ocorrido como substituição total ao agregado natural, e sim como substituição parcial ao cimento e ao agregado natural, os resultados alcançados nesta pesquisa com os resíduos de quartzito demonstram que os valores de resíduos incorporados são superiores aos utilizados por

Tabela 4 - Análise de variância para significância de modelos de regressão.

\begin{tabular}{|c|c|c|c|c|c|c|c|c|c|c|}
\hline $\begin{array}{l}\text { Propriedade } \\
\text { CS }\end{array}$ & $\begin{array}{l}\text { Modelo de } \\
\text { regressão }\end{array}$ & SQR & $\mathrm{GL}$ & QMR & ESQ & $\mathrm{GL}$ & QMR & Teste F & Valor $p$ & $\mathrm{R}^{2}(\%)$ \\
\hline \multirow{3}{*}{ IC } & Linear & 3006,92 & 3 & 1002,31 & 341,77 & 59 & 5,792 & 173,03 & 0,00000 & 89,79 \\
\hline & Quadrática & 251,29 & 6 & 41,88 & 90,48 & 53 & 1,707 & 24,53 & 0,000000 & 97,30 \\
\hline & Cúbica & 29,490 & 2 & 14,745 & 60,990 & 51 & 1,195 & 12,33 & 0,000043 & 98,18 \\
\hline \multirow{3}{*}{ UC } & Linear & 3101,68 & 3 & 1033,89 & 338,88 & 59 & 5,7437 & 180,00 & 0,000000 & 90,15 \\
\hline & Quadrática & 259,51 & 6 & 43,25 & 79,37 & 53 & 1,4976 & 28,88 & 0,000000 & 97,69 \\
\hline & Cúbica & 32,64 & 2 & 16,32 & 46,73 & 51 & 0,9163 & 17,81 & 0,000001 & 98,64 \\
\hline
\end{tabular}

CS: compressão simples; IC: cura por via imersa; UC: cura por via úmida; SQR: soma de quadrados de regressão; GL: graus de liberdade; QMR: quadrados médios de regressão; ESQ: erro significativo dos quadrados; $R^{2}$ : coeficiente de ajuste. 
Cura por via úmida - O\%

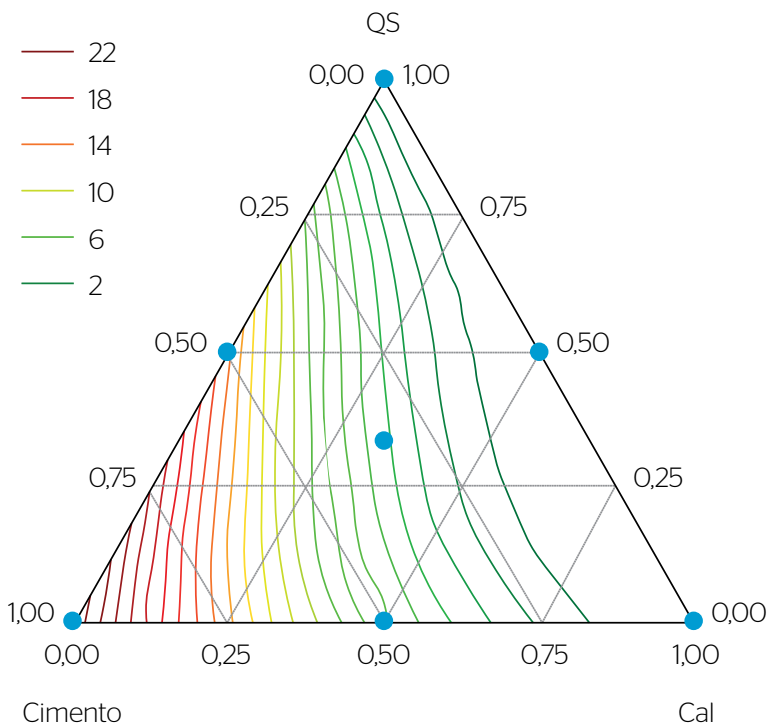

Cimento
Cura por via úmida - 15\%

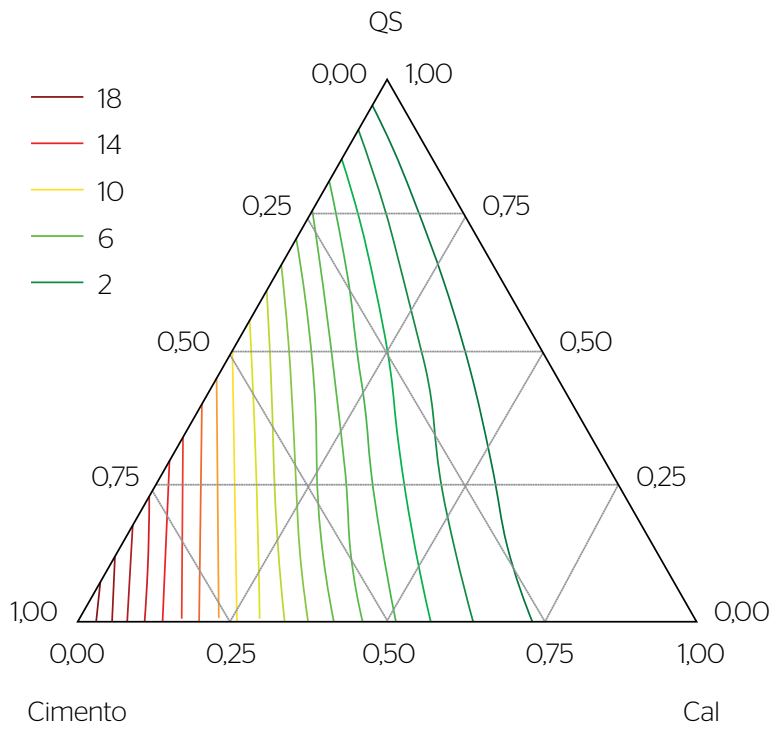

Cura por via úmida - $15 \%$

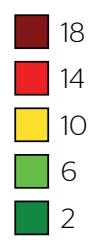

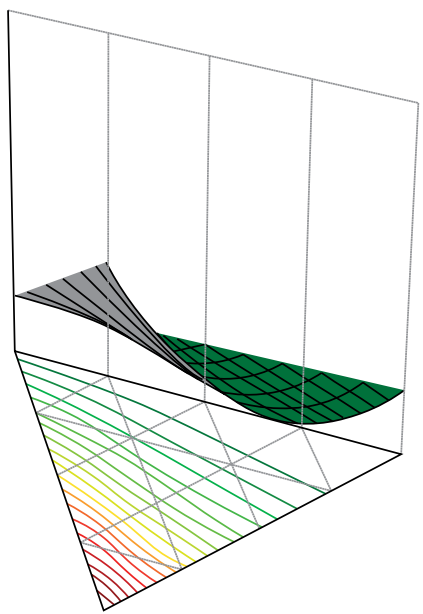

QS: resíduos de fragmentação das aparas.

Figura 2 - Superfícies de resposta para compressão simples por via úmida utilizando 0 e 15\% de resíduos de fragmentação das aparas.

esses autores. Logo, ocorreu um melhor aproveitamento dos resíduos das rochas ornamentais, além de se ter alcançado maiores valores para essa propriedade mecânica, como as composições C e D (Tabela 5), que alcançaram 10,41 e 10,80 $\mathrm{MPa}$, respectivamente.

\section{Potencial toxicológico das argamassas produzidas com os resíduos de quartzito}

$\mathrm{Na}$ escolha das composições mais adequadas para incorporar os resíduos de quartzito e realizar o estudo do potencial toxicológico destas, foi utilizada apenas a UC, em virtude de os resultados obtidos com ela terem sido um pouco melhores que os obtidos com a IC. Logo, com base no modelo de regressão, a Equação 1 foi definida para uma região do delineamento de mistura que pudesse ser transformada em traços usualmente utilizados na construção civil e que apresentasse resistência mecânica suficientemente capaz de atender às especificações técnicas para argamassas de revestimento definidas pela NBR 13281 (ABNT, 2005), isto é, argamassas cuja resistência à CS aos 28 dias mínima fosse $2 \mathrm{MPa}$ ou $\geq 8 \mathrm{MPa}$. Após a definição das composições, determinaram-se experimentalmente os valores previstos para CS-UC, conforme evidenciado na Tabela 5. 
Cura por via imersa - 0\%

QS

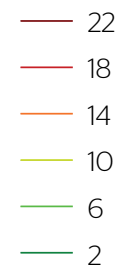$$
0,00
$$$$
0,25
$$

Cimento
Cura por via imersa - 15\%

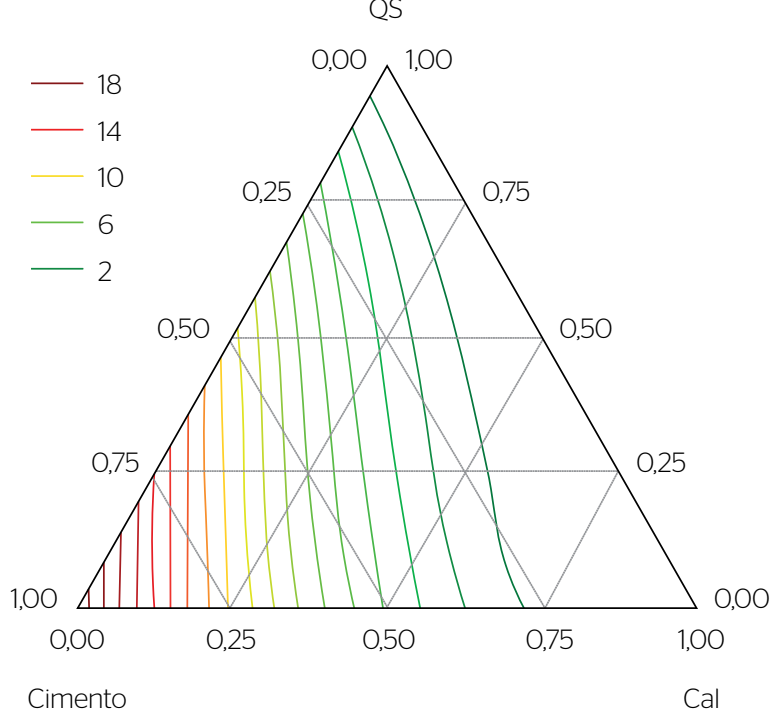

Cura por via imersa - 0\%

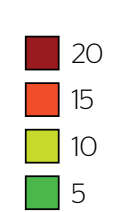

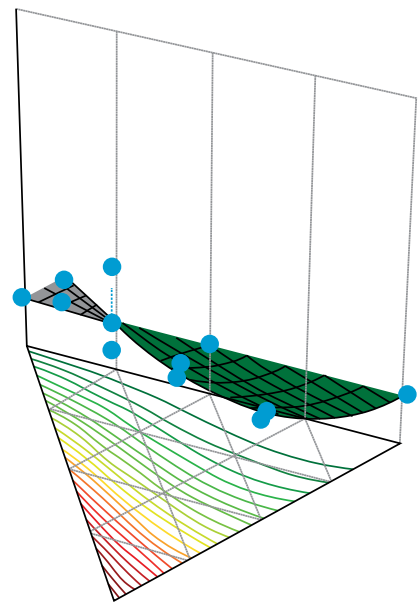

Cura por via imersa - 15\%
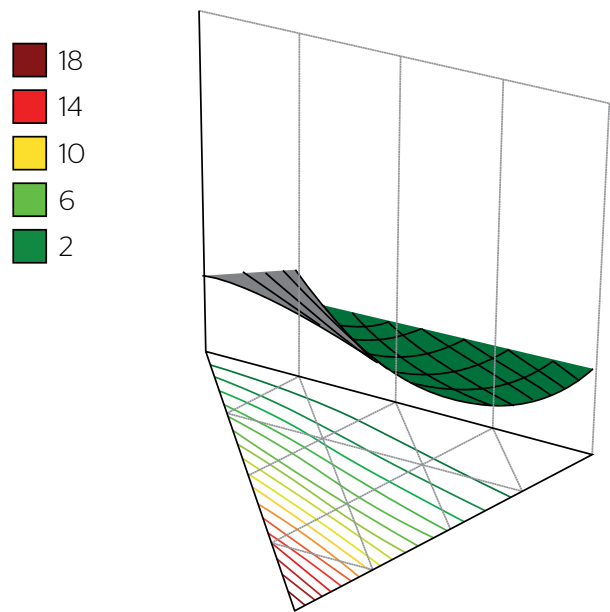

QS: resíduos de fragmentação das aparas.

Figura 3 - Superfícies de resposta para compressão simples por via imersa utilizando 0 e $15 \%$ de resíduos de fragmentação das aparas.

Tabela 5 - Composição de misturas de pontos de verificação, valores medidos correspondentes, valores previstos e intervalo de confiança das resistências à compressão.

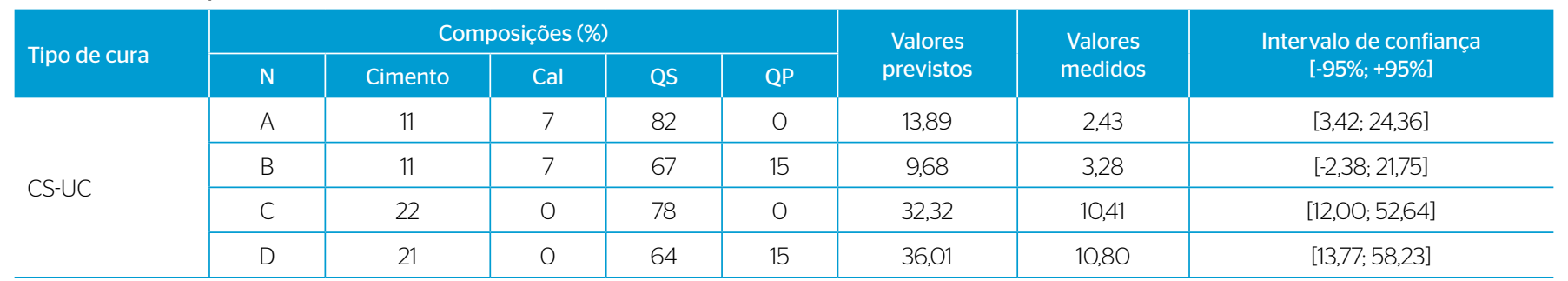

CS-UC: compressão simples por via úmida; QS: resíduos de fragmentação das aparas; QP: resíduos do pó de serragem. 
Pode-se observar que os valores obtidos para as composições escolhidas para desenvolver o estudo do potencial toxicológico estão dentro do intervalo de confiança dos valores previstos, conforme o modelo definido na Equação 1, em todos os casos (Tabela 5).

Os valores obtidos na validação do modelo atenderam às especificações técnicas por terem superado o valor mínimo de $2 \mathrm{MPa}$, determinado pela NBR 13281 (ABNT, 2005) e por autores como Mármol et al. (2010), para resistência à CS aos 28 dias para argamassas de revestimento. Com isso, demonstra-se que é possível obter formulações de argamassas substituindo totalmente o agregado convencional por agregados oriundos dos resíduos de quartzito com resistência adequada, sendo possível incorporar teores elevados de resíduos, superiores a $80 \%$ nas formulações. Com destaque para as composições com teores de até $15 \%$ de resíduo pulverulento, que normalmente é um material difícil de incorporar em formulações por alterar o empacotamento do sistema e que essas adições de finos não alteram a resistência de forma acentuada.

As composições C e D (Tabela 5) são argamassas constituídas apenas de cimento como aglomerante, o que traduziu em melhores resultados de resistência à $\mathrm{CS}$ em relação às composições de matriz cal-cimento (A e B). Apesar disso, as composições com cal obtiveram valores adequados de resistência. Stefanidou, Anastasiou e Filikas (2014) alcançaram resistência de 4,5 MPa aos 28 dias, valor em conformidade com a norma para argamassas constituídas de cimento e $100 \%$ de agregado reutilizado proveniente de resíduos distintos do utilizado nesta pesquisa. Porém, os resultados obtidos com os resíduos de quartzito apresentaram argamassas com valores mais elevados, comprovando a viabilidade técnica de utilização deles no que diz respeito à resistência à compressão.
As composições A, B, C e D foram destorroadas e submetidas a ensaios de lixiviação e solubilização para verificar o potencial toxicológico de liberação de metais pesados de seus resíduos. Os valores encontrados nos seus respectivos extratos lixiviados as classificam como resíduos não perigosos, de acordo com o TCLP 1311 (USEPA, 1992), a NBR 10005 (ABNT, 2004a) e a NBR 10006 (ABNT, 2004b), pois os resultados obtidos estão abaixo dos valores máximos permitidos (Tabela 6).

Os teores obtidos no extrato lixiviado dos resíduos das argamassas confeccionadas com o resíduo de quartzito foram analisados quanto às concentrações de Al, As, Ba, Cd, Cr, Cu, Fe, Mn, Ni e Zn (Tabela 6), e indicaram que os metais presentes nas amostras QS e QP foram imobilizados nas respectivas composições desses compósitos. O controle da lixiviação dos metais pesados em matrizes cimentícias é explicado pela reação química da portlandita $\left(\mathrm{Ca}(\mathrm{OH})_{2}\right)$ e do silicato de cálcio hidratado (C-S-H) com o dióxido de carbono, que formam a calcita $\left(\mathrm{CaCO}_{3}\right)$, proporcionando a imobilização dos metais pesados. Segundo a literatura citada nesta pesquisa, a calcita pode ocorrer por aprisionamento físico (adsorção) ou, ainda, pelas interações químicas (complexação) do contaminante para a matriz de cimento, consequentemente, promovendo a imobilização dele.

Assim como os autores, conseguiu-se imobilizar todos os metais, com destaque para o As, pois apresenta um potencial toxicológico aos sistemas ambientais e danoso à saúde pública maior do que os demais metais analisados. Na matriz cal-cimento (A e B), os resultados obtidos foram melhores que na matriz de cimento ( $\mathrm{C}$ e D), pois estas composições apresentaram $\mathrm{Al}$ acima dos teores máximos permitidos para os extratos lixiviados e solubilizados, mesmo que tenha ocorrido redução significativa da mobilidade deles, quando comparados com os extratos dos resíduos. Os resultados obtidos neste trabalho comprovam a eficácia da reutilização dos resíduos de quartzito em argamassas de

Tabela 6 - Teores determinados e máximos permitidos para o extrato lixiviado e solubilizado das composições de argamassas estudadas.

\begin{tabular}{|c|c|c|c|c|c|c|c|c|c|c|}
\hline \multirow{3}{*}{ Parâmetro } & \multicolumn{5}{|c|}{ Lixiviação } & \multicolumn{5}{|c|}{ Solubilização } \\
\hline & \multicolumn{4}{|c|}{ Teores determinados (mg/L) } & \multirow{2}{*}{$\begin{array}{l}\text { Teores máximos } \\
\text { permitidos (mg/L) }\end{array}$} & \multicolumn{4}{|c|}{ Teores determinados (mg/L) } & \multirow{2}{*}{$\begin{array}{c}\text { Teores máximos } \\
\text { permitidos (mg/L) }\end{array}$} \\
\hline & A & B & C & D & & A & B & C & D & \\
\hline $\mathrm{Al}$ & $<0,10$ & 0,12 & 0,37 & 0,48 & 0,20 & 0,14 & 0,16 & 0,25 & 0,34 & 0,20 \\
\hline As & $<0,01$ & $<0,01$ & $<0,01$ & $<0,01$ & 5,00 & $<0,01$ & $<0,01$ & $<0,01$ & $<0,01$ & 0,01 \\
\hline $\mathrm{Ba}$ & 1,12 & 0,92 & 1,10 & 1,30 & 70,00 & 0,92 & 0,94 & 0,90 & 0,20 & 0,70 \\
\hline $\mathrm{Cd}$ & 0,035 & 0,030 & 0,030 & 0,022 & 0,50 & $<0,01$ & $<0,01$ & $<0,01$ & $<0,01$ & 0,005 \\
\hline $\mathrm{Cr}$ & 0,15 & 0,11 & 0,29 & 0,21 & 5,00 & $<0,01$ & $<0,01$ & $<0,01$ & 0,10 & 0,05 \\
\hline $\mathrm{Cu}$ & 0,024 & 0,016 & 0,016 & 0,014 & 2,00 & 0,013 & $<0,01$ & $<0,01$ & $<0,01$ & 2,00 \\
\hline $\mathrm{Fe}$ & 0,10 & 0,10 & 0,10 & 0,10 & 0,30 & 0,063 & 0,045 & 0,040 & 0,038 & 0,30 \\
\hline $\mathrm{Ni}$ & 0,13 & 0,081 & 0,067 & 0,050 & 7,00 & 0,029 & 0,031 & 0,012 & $<0,01$ & 0,07 \\
\hline $\mathrm{Mn}$ & 0,035 & 0,029 & 0,024 & 0,012 & 0,40 & $<0,01$ & $<0,01$ & $<0,01$ & $<0,01$ & 0,10 \\
\hline $\mathrm{Zn}$ & 0,030 & 0,031 & 0,016 & 0,015 & 5,00 & 0,012 & $<0,01$ & $<0,01$ & $<0,01$ & 5,00 \\
\hline
\end{tabular}


cal-cimento ou de cimento, uma vez que ocorreu a redução da lixiviação dos metais pesados presentes nos resíduos e, principalmente, a imobilização quase que completa do As.

\section{CONCLUSÃO}

As conclusões desta pesquisa são:

- Os QS e QP são classificados como Resíduos Classe I — Perigoso (NBR 10004), uma vez que apresentaram metais pesados em extratos lixiviados e solubilizados superiores aos limites das normas técnicas;

- O melhor modelo de regressão encontrado foi o da condição da UC, pois forneceu maiores valores de resistência à CS aos 28 dias para um nível de significância de 5\%, com maior valor de $\mathrm{R}^{2}$ e $\mathrm{F}$ calculado $(17,81)$ oito vezes maior que o $\mathrm{F}$ tabulado;

- Os valores das resistências à CS aos 28 dias alcançados para as argamassas confeccionadas com os resíduos de quartzito em substituição total ao agregado natural neste estudo confirmam a viabilidade técnica da utilização deles como matéria-prima para argamassas quanto ao aspecto dessa propriedade mecânica, uma vez que a resistência foi superior a $2 \mathrm{MPa}$;

- Os resíduos das argamassas confeccionadas com QS e QP são classificados ambientalmente como resíduos não perigosos, sendo as composições C e D não inertes, porque apresentaram o teor do extrato lixiviado do $\mathrm{Al}$ reduzido, porém um pouco maior que o máximo permitido;

- A reutilização dos resíduos de quartzito por meio da sua incorporação em argamassas imobilizou o As e outros metais presentes nesses resíduos, comprovando a eficiência da reutilização desses em matrizes de cimento e cal-cimento como técnica de imobilização dos íons de metais pesados, de modo a promover uma alternativa tecnologicamente viável e ambientalmente adequada para os resíduos oriundos da exploração de quartzitos.

\section{REFERÊNCIAS}

AGRAFIOTI, E.; KALDERIS, D.; DIAMADOPOULOS, E. (2014) Arsenic and chromium removal from water using biochars derived from rice husk. Organic solid wastes and sewage sludge. Journal of Environmental Management, v. 133, p. 309-314. https://doi. org/10.1016/j.jenvman.2013.12.007

AMERICAN SOCIETY FOR TESTING AND MATERIALS (ASTM). (2008) ASTM C230/ C 230M-08: Standard Specification for Flow Table for Use in Tests of Hydraulic Cement. United States: ASTM.

ANDRÉ, A.; BRITO, J.; ROSA, A.; PEDRO, D. (2014) Durability performance of concrete incorporating coarse aggregates from marble industry waste. Journal of Cleaner Production, v. 65, p. 389-396. https://doi.org/10.1016/j.jclepro.2013.09.037

ARUKWE, A.; EGGEN, T.; MÖDER, M. (2012) Solid waste deposits as a significant source of contaminants of emerging concern to the aquatic and terrestrial environments - A developing country case study from Owerri, Nigeria. Science of the Total Environment, v. 438, p. 94-102. https://doi.org/10.1016/j.scitotenv.2012.08.039

ASSOCIAÇÃO BRASILEIRA DE NORMAS TÉCNICAS (ABNT). (1996) NBR 7215: Cimento Portland - Determinação da resistência à compressão. Rio de Janeiro: ABNT.

ASSOCIAÇÃO BRASILEIRA DE NORMAS TÉCNICAS (ABNT). (2002) NBR 13276: Argamassa para Assentamento e Revestimento. Rio de Janeiro: ABNT.

ASSOCIAÇÃO BRASILEIRA DE NORMAS TÉCNICAS (ABNT). (2004a) NBR 10004: Resíduos sólidos - Classificação. Rio de Janeiro: ABNT.
ASSOCIAÇÃO BRASILEIRA DE NORMAS TÉCNICAS (ABNT). (2004b) NBR 10005: Procedimento para obtenção de extrato lixiviado de resíduos sólidos. Rio de Janeiro: ABNT.

ASSOCIAÇÃO BRASILEIRA DE NORMAS TÉCNICAS (ABNT) (2004c) NBR 10006: Procedimento para obtenção de extrato solubilizado de resíduos sólidos. Rio de Janeiro: ABNT.

ASSOCIAÇÃO BRASILEIRA DE NORMAS TÉCNICAS (ABNT). (2005) NBR 13281: Argamassa para assentamento e revestimento de paredes e tetos - Requisitos. Rio de Janeiro: ABNT.

BACARJI, E.; TOLEDO FILHO, R.D.; KOENDERS, E.A.B.; FIGUEIREDO, E.P.; LOPES, J.L.M.P. (2013) Sustainability perspective of marble and granite residues as concrete fillers. Construction and Building Materials, v. 45, p. 1-10. https://doi.org/10.1016/j. conbuildmat.2013.03.032

BOX, G.E.P.; WETZ, J. (1973) Criteria for judging adequacy of estimation by an aproximate response funtion. University of Wisconsis Technical Report 9. Wisconsis: University of Wisconsis.

BRAGA, M.; BRITO, J.; VEIGA, R. (2012) Incorporation of fine concrete aggregates in mortars. Construction and Building Materials, v. 36, p. 960-968. https://doi.org/10.1016/j.conbuildmat.2012.06.031

BRASIL. (1981) Lei Federal no 6.938, de 31 de agosto de 1981 Dispõe sobre a Política Nacional do Meio Ambiente, seus fins e mecanismos de formulação e aplicação, e dá outras providências. Diário Oficial da União, Brasília. Disponível em: <http://www.planalto. gov.br/ccivil_O3/leis/L6938.htm>. Acesso em: 19 dez. 2017. 
BRASIL. (1988) Constituição da República Federativa do Brasil. Texto constitucional promulgado em 5 de outubro de 1988, com as alterações adotadas pelas Emendas Constitucionais nos 1/1992 a 76/2013, pelo Decreto Legislativo no 186/2008 e pelas Emendas Constitucionais de Revisão nos 1 a 6/1994. 40.ed. com índice. Brasília: Centro de Documentação e Informação (CEDI), 2013. 464 p. Disponível em: <http://www2.camara.leg.br/atividade-legislativa/ legislacao/Constituicoes_Brasileiras/constituicao1988.html>. Acesso em: 20 mar. 2018

BRASIL. (2010) Lei Federal no 12.305, de 2 de agosto de 2010. Institui a Política Nacional de Resíduos Sólidos; altera a Lei no 9.605, de 12 de fevereiro de 1998; e dá outras providências. Diário Oficial da União, Brasília. Disponível em: <http://www.mma.gov.br/port/ conama/legiabre.cfm?codlegi=636>. Acesso em: 12 jan. 2018.

CAMARINI, G.; SOARES, M.S. (2018) Propriedades de argamassas de cimento produzidas com resíduo de isolador de porcelana. Matéria, v. 23, n. 1. https://doi.org/10.1590/s1517-707620170001.0302

CORINALDESI, V.; MORICONI, G.; NAIK, T.R. (2010) Characterization of marble powder for its use in mortar and concrete. Construction and Building Materials, v. 24, n. 1, p. 113-117. https://doi.org/10.1016/j. conbuildmat.2009.08.013

COUSSY, S.; BENZAAZOUA, M.; BLANC, D.; MOSZKOWICZ, P.; BUSSIĖRE, B. (2012) Assessment of arsenic immobilization in synthetically prepared cemented paste backfill specimens. Journal of Environmental Management, v. 93, n. 1, p. 10-21. https://doi. org/10.1016/j.jenvman.2011.08.015

DANTAS, G.C.B.; FORBELONI, J.V.; PACHECO, A.S.V.; CAMPELO, I.C.; FARIAS, H.M. (2017) Perceptions of waste pickers in an association located in Seridó/RN about the adverse working conditions: an analysis of the collective subject. Revista Eletrônica em Gestão, Educação e Tecnologia Ambiental, v. 21, n. 3, p. 210-221. https://doi. org/10.5902/2236117028013

ERCIKDI, B.; KULEKCI, G.; YILMAZ, T. (2015) Utilization of granulated marble wastes and waste bricks as mineral admixture in cemented paste backfill of sulphide-rich tailings. Construction and Building Materials, v. 93, p. 573-583. https://doi.org/10.1016/j. conbuildmat.2015.06.042

ERGÜN, A. (2011) Effects of the usage of diatomite and waste marble powder as partial replacement. Construction and Building Materials, v. 25, n. 2, p. 806-812. https://doi.org/10.1016/j. conbuildmat.2010.07.002

GAMEIRO, F.; BRITO, J.; SILVA, D.C. (2014) Durability performance of structural concrete containing fine aggregates from waste generated by marble quarrying industry. Engineering Structures, v. 59, p. 654-662. https://doi.org/10.1016/j.engstruct.2013.11.026

GERVEN, T.V.; BAELEN, D.V.; DUTRÉ, V.; VANDECASTEELE, C. (2004) Influence of carbonation and carbonation methods on leaching of metals from mortars. Cement and Concrete Research, v. 34, n. 1, p. 149-156. https://doi.org/10.1016/S0008-8846(03)00255-2

GUILLÉN, J.; TEJADO, J.J.; BEAZA, A.; SALAS, A.; MUÑOZ-MUÑOZ, J.G. (2O14) Environmental impact of a granite processing factory as source of naturally occurring radionuclides. Applied Geochemistry, v. 47 , p. $122-129$

HAMEED, M.; SEKAR, A. (2009) Properties of green concrete containing quarry rock dust and marble sludge powder as a fine aggregate. Journal of Engineering and Applied Sciences, v. 4, n. 4, p. 83-89.

JIMÉNEZ, J.R.; AYUSO, J.; LÓPEZ, M.; FERNÁNDEZ, J.M.; BRITO, J. (2O13) Use of fine recycled aggregates from ceramic waste in masonry mortar manufacturing. Construction and Building Materials, v. 40, p. 679-690. https://doi.org/10.1016/j.conbuildmat.2012.11.036

JUNKES, J.A.; PRATES, P.B.; HOTZA, D.; SEGADÃES, A.M. (2O12) Combining mineral and clay-based wastes to produce porcelainlike ceramics: An exploratory study. Applied Clay Science, v. 69, p. 50-57. https://doi.org/10.1016/j.clay.2012.08.009

MÁRMOL, I.; BALLESTER, P.; CERRO, S.; MONRÓS, G.; MORALES, J.; SÁNCHEZ, L. (2010) Use of granite sludge wastes for the production of coloured cement-based mortars. Cement \& Concrete Composites, v.32, n. 8, p.617-622.https://doi.org/10.1016/j.cemconcomp.2010.06.003

MARTínEZ, I.; ETXEBERRIA, M.; PAVÓN, E.; DIAZ, N. (2013) A comparative analysis of the properties of recycled and natura aggregate in masonry mortars. Construction and Building Materials, v. 49, p. 384-392. https://doi.org/10.1016/j.conbuildmat.2013.08.049

MEHTA, P.K.; MONTEIRO, P.J.M. (2008) Concreto, Microestrutura, Propriedades e Materiais. 3a ed. São Paulo: IBRACON.

MERSONI, C.; REICHERT, G.A. (2017) Comparação de cenários de tratamento de resíduos sólidos urbanos por meio da técnica da Avaliação do Ciclo de Vida: o caso do município de Garibaldi, RS. Engenharia Sanitária e Ambiental, v. 22, n. 5, p. 863-875.

MOREIRA, M.S.; MANHÃES, J.P.V.T; HOLANDA, J.N.F. (2008) Processing of red ceramic using ornamental rock powder waste. Journal of 'Materials Processing Technology, v. 196, n. 1-3, p. 88-93. https://doi.org/10.1016/j.jmatprotec.2007.05.008

NAKWANIT, S.; VISOOTTIVISETHA, P.; KHOKIATTIWONG, S. SANGCHOOM, W. (2011) Management of arsenic-accumulated waste from constructed wetland treatment of mountain tap water. Journal of Hazardous Materials, v. 185, n. 2-3, p. 1081-1085. https://doi. org/10.1016/j.jhazmat.2010.10.017

PATEL, V.N.; SHAH, N.D. (2015) Suitability of Porcelain and Marble Industrial Waste Powder to Produce High Performance Concrete. American Journal of Civil Engineering and Architecture, v. 3, n. 3 , p. 59-63. https://doi.org/10.12691/ajcea-3-3-1

PIMENTEL, L.L.; PISSOLATO JÚNIOR, O.; JACINTHO, A.E.P.G.A.; MARTINS, H.L.S. (2018) Argamassa com areia proveniente da britagem de resíduo de construção civil - Avaliação de características físicas e mecânicas. Matéria, v. 23, n. 1, e-11969. https://doi.org/10.1590/s1517-707620170001.0305

RAMOS, T.; MATOS, A.M.; SCHMIDT, B.; RIO, J.; SOUSA-COUTINHO J. (2013) Granitic quarry sludge waste in mortar: Effect on strength and durability. Construction and Building Materials, v. 47, p. 1001-1009. https://doi.org/10.1016/j.conbuildmat.2013.05.098 
RIBEIRO, V.A.S.; SILVA, A.L.; SILVA, M.R.; DIAS, J.C. (2007) Reciclagem de escória cristalizada para a produção de argamassa. Matéria, v. 12, n. 2, p. 339-345. https://doi.org/10.1590/ S1517-70762007000200012

SAJEDI, F.; RAZAK, H.A. (2011) Effects of curing regimes and cement fineness on the compressive strength of ordinary Portland cement mortars. Construction and Building Materials, v. 25, n. 4, p. 2036-2045. https://doi.org/10.1016/j. conbuildmat.2010.11.043

SCHACKOW, A.; STRINGARI, D.; SENFF, L.; CORREIA, S.L.; SEGADÃES, A.M. (2015) Influence of fired clay brick waste additions on the durability of mortars. Cement \& Concrete Composites, v. 62 , p. 82-89. https://doi.org/10.1016/j.cemconcomp.2015.04.019

SHARMA, A.K.; TJELL, J.C.; SLOTH, J.J.; HOLM, P.E. (2O14) Review of arsenic contamination exposure through water and food and low cost mitigation options for rural areas. Applied Geochemistry, v. 41, p. 11-33. https://doi.org/10.1016/j.apgeochem.2013.11.012

STEFANIDOU, M.; ANASTASIOU, E.; FILIKAS, K.G. (2014) Recycled sand in lime-based mortars. Waste Management, v. 34, п. 12, p. 2595-2602. https://doi.org/10.1016/j.wasman.2014.09.005

SUKHOLTHAMAN, P.; SHIRAHADA, K. (2015) Technological challenges for effective development towards sustainable waste management in developing countries: Case study of Bangkok
Thailand. Technology in Society, v. 43, p. 231-239. https://doi. org/10.1016/j.techsoc.2015.05.003

SULLIVAN, C.; TYRER, M.; CHEESEMAN, C.R.; GRAHAM, N.J.D. (2O10) Disposal of water treatment wastes containing arsenic: A review. Science of the Total Environment, v. 408, n. 8, p. 1770-1778. https:// doi.org/10.1016/j.scitotenv.2010.01.010

TENNICH, M.; KALLEL, A.; OUEZDOU, M.B. (2015) Incorporation of fillers from marble and tile wastes in the composition of selfcompacting concretes. Construction and Building Materials, v. 91, p. 65-70. https://doi.org/10.1016/j.conbuildmat.2015.04.052

TORRES, P.; FERNANDES, H.R.; OLHERO, S.; FERREIRA, J.M.F. (2009) Incorporation of wastes from granite rock cutting and polishing industries to produce roof tiles. Journal of the European Ceramic Society, v. 29, n. 1, p. 23-30. https://doi.org/10.1016/j. jeurceramsoc.2008.05.045

TOUJAGUEZ, R.; ONO, F.B.; MARTINS, V.; CABRERA, P.P.; BLANCO, A.V.; BUNDSCHUH, J.; GUILHERME, L.R.G. (2O13) Arsenic bioaccessibility in gold mine tailings of Delita Cuba. Journal of Hazardous Materials, v. 262, p. 1004-1013. https://doi.org/10.1016/j.jhazmat.2013.01.045

UNITED STATES ENVIRONMENTAL PROTECTION AGENCY (USEPA). (1992) TCPL 1311: test methods for evaluating solid waste, toxicity characteristics leaching procedure. Physical/Chemical methods. SW-846. Estados Unidos: USEPA. 